\section{Kognitive Störungen bei Multipler Sklerose}

Pasquale Calabrese

Neurologische Universitätsklinik (Knappschaftskrankenhaus) Bochum

psychoneuro 2003; 29 (7): 344-348

Die Multiple Sklerose (MS) ist eine der häufigsten neurologischen Erkrankungen des frühen und mittleren Erwachsenenalters. Sie birgt für die Betroffenen neben den körperlichen Beeinträchtigungen auch viele psychosoziale Probleme. Bei nahezu 50\% der Patienten treten im Verlauf dieser Erkrankung kognitive Defizite auf. Sie betreffen zumeist die Bereiche der Aufmerksamkeit, des Gedächtnisses und der geistigen Flexibilität. Diese Störungen können die Patienten sowohl im Arbeitsleben als auch in ihren sozialen Aktivitäten beeinträchtigen. Obgleich neuere Studien den hohen Vorhersagewert der kognitiven Leistungsfähigkeit hinsichtlich des Verbleibs in der Arbeitswelt herausstellen, finden erst in jüngster Zeit die MS-bedingten Hirnleistungsstörungen zunehmende Berücksichtigung sowohl in der Gesamteinschätzung des Krankheitsbildes als auch in der Effizienzbeurteilung von modernen Pharmakotherapien. In dieser Arbeit wird auf die Erfassung der relevanten Defizite und auf deren Alltagsrelevanz eingegangen. Schließlich wird ein funktionell-neuroanatomisches Modell, welches die organische Basis der kognitiven Defizite erklären könnte, vorgestellt.

$\mathrm{D}$ ie Multiple Sklerose (MS) gilt als eine der häufigsten neurologischen Erkrankungen des frühen und mittleren Erwachsenenalters. Nach derzeitigem Kenntnisstand kann die MS ätiopathogenetisch als autoimmunvermittelte Erkrankung des Zentralnervensystems angesehen werden, die zu multiplen, zeitlich und örtlich disseminierten Demyelinisierungen unterschiedlichster Lokalisationen führt. Der bei den meisten Erkrankungsfällen anfänglich schubförmige Krankheitsverlauf (schubförmige MS) kann in der weiteren Krankheitsevolution in einen chronisch-progredienten Verlauf übergehen. Das Erstmanifestationsalter liegt meist zwischen dem 20. und 50. Lebensjahr, mit einem Gipfel in der dritten Lebensdekade, wobei das weibliche Geschlecht überwiegt. Somit fällt der Krankheitsausbruch für die meisten Patienten in die Lebensperiode der beruflichen und sozialen Etablierung und stellt einen Großteil der Betroffenen vor verschiedenste psychosoziale Probleme. Neben multip- len neurologischen Dysfunktionen können im Verlauf der MS unterschiedlichste neuropsychologische können als isolierte Erstsymptome auftreten und/oder im Krankheitsverlauf exazerbieren (2). Gerade wegen der zunehmenden Technologisierung der Lebens- und Arbeitsbedingungen ist (neben dem physischen Behinderungsgrad) insbesondere die kognitive Leistungsfähigkeit ein relevanter Faktor geworden, der im Einzelfall über den Verbleib im Erwerbsleben entscheidet (3). Darüberhinaus stellt die kognitive Funktionstüchtigkeit aber auch für die Teilnahme des Einzelnen am sozialen Leben eine wichtige Bedingung dar und ist damit für die Lebensqualität von entscheidender Bedeutung (4). Die Beschreibung kognitiver Störungen bei MS-Patienten findet sich schon in der historischen neurologischen Literatur. Während zu Beginn des Jahrhunderts die kognitiven Leistungsdefizite unter dem Oberbegriff der Demenz subsumiert wurden (organische WesensStörungen imponieren (1). Diese

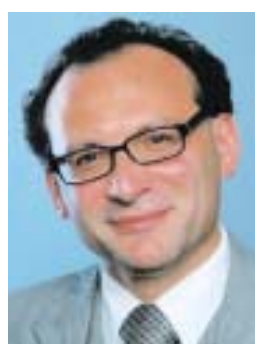

Pasquale Calabrese änderungen im Ausmaße einer globalen Demenz sind nur in vereinzelten, meist fortgeschritteneren Stadien einer progredienten MS anzutreffen), sind die MS-bedingten kognitiven Defizite erst in den letzten beiden Jahrzehnten einer eingehenden Systematisierung unterzogen worden. Hierbei wurde deutlich, dass in der Mehrheit der MS-Fälle und in Abhängigkeit vom Verlaufstyp eine Symptomkonstellation distinkter neuropsychologischer Teilleistungsbeeinträchtigungen dominiert. Aktuelle Studien gehen hierbei von einer Auftretenshäufigkeit neuropsychologischer Defizite bei 50-70\% der MS-Patienten aus, wobei Aufmerksamkeits- und Gedächtnisstörungen sowie Beeiträchtigungen der kognitiven Flexibilität (i.S. eines Dysexekutivsyndroms) zu den häufigsten Beeinträchtigungen zählen (5). In Abhängigkeit von der zerebralen Beteiligung sind aber auch global-intellektuelle Nivellierungen in Verbindung mit psychiatrisch relevanten Störungen anzutreffen, die insgesamt einen multiprofessionellen Behandlungsansatz erfordern (6).

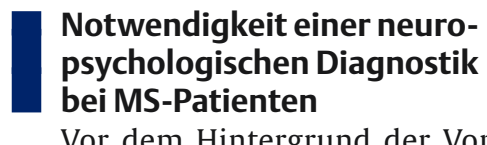

Vor dem Hintergrund der Vorkommenshäufigkeit und Alltagsrelevanz kognitiver Störungen sollte eine klinisch-neuropsychologische Untersuchung immer Bestandteil einer Gesamtdiagnostik bei Patienten mit Multipler Sklerose sein. Die Überprüfung der kognitiven Leistungsfähigkeit sollte mittels stan- 
dardisierter und normierter neuropsychologischer Testverfahren erfolgen (Tab. 1). Ergänzend hierzu können zur Einschätzung und Objektivierung affektiv-emotionaler Beeinträchtigungen auch standardisierte Fragebögen sowie strukturierte Interviews eingesetzt werden. Da insbesondere Aufmerksamkeit, Gedächtnis und mentale Flexibilität bereits in Frühphasen der Erkrankung gegenüber anderen Partialleistungen deutlich beeinträchtigt sein können, sollte eine kognitive Leistungseinschätzung diese Leistungsaspekte besonders berücksichtigen. Oft sind die Zeitgrenzen in der ärztlichen Routinediagnostik sehr eng gesetzt und nicht immer stehen neuropsychologisch ausgebildete Fachkollegen zur Verfügung, die eine eingehendere Untersuchung der kognitiven Leistungen gewährleisten können. Dementsprechend ist einerseits die Entwicklung von sog. Screening-Verfahren, d.h. zeitökonomischer Globalverfahren, die innerhalb enger Zeitgrenzen eine primäre Einschätzung der kognitiven Leistungsfähigkeit erlauben, zu fordern. Genauso wichtig ist dann, dass sich bei positivem Resultat eines solchen Screenings eine qualifizierte neuropsychologische Untersuchung zur genaueren Beschreibung (mit möglichen Behandlungsempfehlungen) der Defizite anschließt. Diese kann von klinischen Neuropsychologen geleistet werden. Im Folgenden werden einige, insbesondere zu dem ge- nannten Partialleistungen herausgearbeitete Befunde referiert.

\section{Aufmerksamkeits- und Konzentrationsleistungen}

Die Fähigkeit der kurzfristigen oder überdauernden, diffusen Aufmerksamkeitssteigerung oder zielgerichteten Aufmerksamkeitszuwendung bildet das Grundgerüst für die kognitive Informationsverarbeitung. Zahlreiche klinische und experimentelle Studien legen eine Unterteilung in verschiedene Aufmerksamkeitsbereiche nahe (selektive Aufmerksamkeit, geteilte Aufmerksamkeit, Aktiviertheit, Vigilanz). Hierbei können verschiedene Aufmerksamkeitskomponenten je nach Lateralität, Lokalisation und Ausdehnung einer Hirnschädigung in unterschiedlichem Ausmaß beeinträchtigt werden. Bei MS-Patienten finden sich insbesondere Störungen im Bereich der komplexen Aufmerksamkeitsleistungen (2), während die Vigilanzleistungen relativ unbeeinträchtigt sind. Kognitives Tempo und geteilte Aufmerksamkeit sind im längsschnittlichen Verlauf zunehmend betroffen (8) und weisen einen Zusammenhang zu Schubaktivität und Läsionsvolumen auf. Nach neuesten Studien sind insbesondere die kortikale Atrophie sowie das temporale, occipitale und frontale Läsionsvolumen eng mit Aufmerksamkeitsstörungen assoziiert (8). In der klinischen Praxis werden zur Erfassung sog. „Attentionalitätsleis- tungen“ sowohl Papier- und Bleistifttests als auch computerisierte Testverfahren eingesetzt (s. Tab. 1). Gerade wegen der „motorischen Kontaminierung“ der erstgenanten Methode und wegen der Beschränkung auf die visuomotorisch-visuokonstruktive Dimension sind computerisierte Verfahren mit der Möglichkeit verschiedener Varianten der Reizdarbietung (akustisch und/oder visuell) vorzuziehen. Hier sei die Testbatterie zur Aufmerksamkeitsprüfung (TAP) besonders empfohlen, da mit diesem Testverfahren verschiedene Aufmerksamkeitskomponenten mittels einfacher Reaktionsanforderungen erfasst werden können. Bei Sicherstellung einer ausreichenden sensomotorischen Funktionsintegrität können aber auch einfache Verfahren - wie der d2-Aufmerksamkeits-Belastungstest oder der Trail-Making-Test (TMT) eingesetzt werden. Die Interpretation zeitkritischer Verfahren (wie z.B. dem PASAT) erfordert seitens des Untersuchers ein hohes Maß an Durchführungsroutine. Darüber hinaus sei gerade bei zeitlimitierten Verfahren mit verbaler Antworterfordernis auf die durch eine Artikulationsstörung (z.B. schlaffe Dysarthrie verursachte Testverzerrung hingewiesen.

\section{Sprache und Intelligenzleistungen}

Wenngleich bei vielen MS-Patienten mit bulbärem Befall Artikula-

\section{Tab. 1 Testverfahren}

\begin{tabular}{|c|c|c|}
\hline Bereich & Testverfahren & Bemerkungen \\
\hline $\begin{array}{l}\text { Aufmerksamkeit } \\
\text { (selektive Aufmerksamkeit, } \\
\text { geteilte Aufmerksamkeit, } \\
\text { Aktiviertheit, Vigilanz) }\end{array}$ & $\begin{array}{l}\text { d2, TMT-A, PASAT, } \\
\text { TAP }\end{array}$ & $\begin{array}{l}\text { - „motorische Kontamination“ beachten } \\
\text { - Artikulationsstörungen bei zeitabhängigen, verbalen Tests } \\
\text { berücksichtigen } \\
\text { - Visus und Akusis }\end{array}$ \\
\hline $\begin{array}{l}\text { Gedächtnis } \\
\text { (Kurzzeit-, Langzeit-, explizites } \\
\text { und implizites Gedächtnis) }\end{array}$ & $\begin{array}{l}\text { VLMT, GNL, VVM, } \\
\text { VLT/NVLT }\end{array}$ & $\begin{array}{l}\text { - Materialspezifische Unterschiede (verbal vs. visuell) } \\
\text { beachten } \\
\text { - Kurz- u. längerfristige Behaltensintervalle prüfen } \\
\text { - „Lernkurve“ beurteilen (Inkrement, Stabilität) }\end{array}$ \\
\hline $\begin{array}{l}\text { Exekutivfunktionen } \\
\text { (Handlungsplanung, Flexibilität, } \\
\text { Handlungsausführung, Strategien) }\end{array}$ & $\begin{array}{l}\text { TMT-B, WCST, BADS, } \\
\text { TEA, TKS }\end{array}$ & $\begin{array}{l}\text { - Durchführungsstringenz beachten (z.B. Wiederholungen, } \\
\text { Perseverationstendenzen, Interferenzanfälligkeit) } \\
\text { - „Alltagsverifikation“ }\end{array}$ \\
\hline
\end{tabular}

Abkürzungen: BADS= Behavioural Assessment of the Dysexecutive Syndrome; $\mathrm{d} 2=\mathrm{d} 2$-Aufmerksamkeits-Belastungs-Test; $\mathrm{GNL}=\mathrm{Gesichter}$ Namen-Lerntest; PASAT=Paced Auditory-Serial-Addition-Test; TAP= Testbatterie zur Aufmerksamkeitsprüfung; TEA=Test of Everyday-Attention; TMT=Trail-Making-Test; TKS=Test zum kognitiven Schätzen; VLMT=Verbaler Lern- u. Merkfähigkeitstest; VVM= Visueller und Verbaler Merkfähigkeitstest; WCST=Wisconsin-Card-Sorting-Test. (Alle Verfahren finden sich im Testkatalog der Testzentrale/Göttingen bzw. Fa. PSYTEST [Fa. Vera Fimm, Würselen] für die TAP. 
tionsprobleme auftreten können, sind Sprachstörungen i.S. von Aphasien bei MS-Patienten eher selten. Sind jedoch sprachrelevante, kortiko-subkortikale Hirnregionen betroffen (zumeist linksseitige, perisylvische Herde mit Beteiligung der Arcuatusregion und/oder extensiven Demyelinisierungen der anterioren, periventrikulären Region), können neben den Artikulationsstörungen auch Sprachstörungen auftreten. Da aphasische Syndrome jedoch bei MS-Patienten eher eine Ausnahme sind, können unauffällige Leistungen in verbal orientierten Testverfahren von bestehenden kognitiven Defiziten ablenken. Dies bedeutet, dass die intellektuellen Funktionen im Querschnitt durch Verballeistungen allein nicht ausreichend beschrieben werden können. Erst in der längsschnittlichen Betrachtung kommt es zu einem allgemeinen Absinken der gesamtintellektuellen (auch der verbalen) Leistung. In einer aktuellen Longitudinalstudie konnte der Stellenwert sog. „kritischer Leistungsdifferenzen“" (d.h. über eine statistisch zu erwartende Wahrscheinlichkeit hinausgehende Leistungsdiskrepanzen zwischen verschiedenen Subtests bei einem Individuum) herausgestellt werden (8). Praktisch bedeutet dies, dass der Interpretationsschwerpunkt

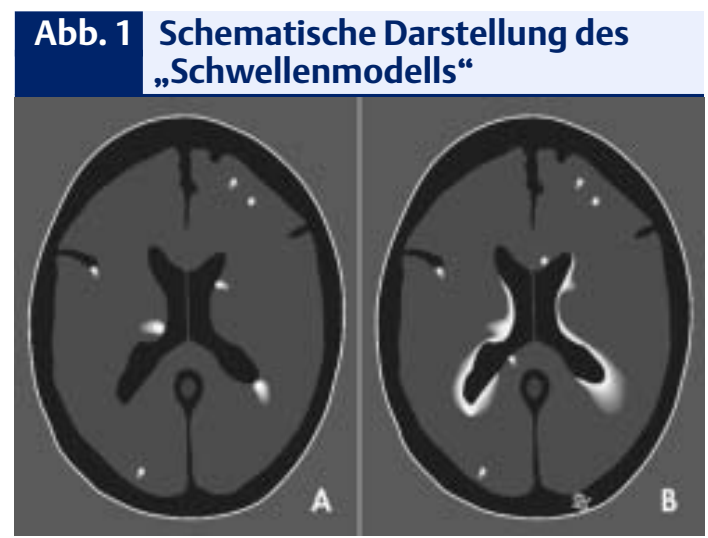

Dargestellt sind unterschiedliche zerebrale Befallsmuster im Rahmen einer MS. Während Läsionsmuster A (je nach Lokalisation der Demyelinisierungsherde) zu distinkten neuropsychologischen Teilleistungsstörungen führen kann, mündet Befallsmuster $B$ aufgrund der topografisch multiplen Diskonnektionen in eine globale, kognitive Leistungsbeeinträchtigung (aus: Calabrese P. in Sturm W\& Wallesch CW (Hrsg.) Lehrbuch der klinischen Neuropsychologie, Amsterdam: Swets \& Zeitlinger, 2000: 569). von Intelligenztests auf den Vergleich von Leistungsminima und maxima gelegt werden sollte. Neben einer „Profildarstellung“, die auch unter (Berufs-)Beratungsaspekten eine genauere Einordnung und $\mathrm{Ge}-$ wichtung des Leistungsspektrums erlaubt, lassen sich durch diese Subtestvergleiche relevante Defizitmuster aufdecken, die durch eine Globalbeurteilung eines Gesamttests (in Form einer IQ-Angabe) verschleiert werden. Für einige, im deutschsprachigen Raum etablierte Verfahren (z.B. Hamburg-Wechsler-Intelligenztest für Erwachsene, HAWIE) stehen Kennwerte zur Verfügung, die einen kritischen Leistungsvergleich auf Individualebene erlauben.

\section{Mentale Flexibilität und Problemlösefähigkeit}

Unter mentaler Flexibilität wird die Fähigkeit verstanden, kognitive Ressourcen stringent, zielorientiert und flexibel zur Lösung eines bestimmten Problems nutzen zu können. Hierbei reicht das Spektrum vom Lösen einer abstrakten Rechenaufgabe bis zur Planung und Durchführung einer Alltagshandlung ( $\mathrm{Zu}-$ bereitung einer Speise, Planung einer Reise etc.). Aus der Komplexität der an diese Fähigkeit gestellten Anforderung wird deutlich, dass sowohl Aufmerksamkeits- als auch Gedächtnisaspekte hieran beteiligt sind. Zwar entbehren die zur Überprüfung dieser Fähigkeit eingesetzten Verfahren (häufig Kategorisierungs- und Sortieraufgaben wie der Wisconsin Card Sorting Test (WCST) und der Tower-of-Hanoi Test) jegliche „ökologische“ Relevanz; jedoch werden in diesen Verfahren die zur erfolgreichen Problemlösung notwendigen Komponenten (Handlungsplanung, Selektion der notwendigen Schritte, Konzeptwechsel etc.) erfasst. Insgesamt zeigt der systematische Einsatz von Problemlöse- und Flexibilitätstests, dass die Leistungsgüte bei MS-Patienten vom zerebralen, frontalen Befallsmuster und weniger vom Gesamtläsionsvolumen abhängt (9).

\section{Gedächtnisstörungen bei MS}

Die Häufigkeit von Gedächtnisstörungen bei MS-Patienten wird in der Literatur mit 40-50\% angegeben. Bei den meisten Studien wird das Kurzzeitgedächtnis bei MS-Patienten als „nicht beeinträchtigt“ herausgestellt. Dagegen stellen sich in vielen Untersuchungen sowohl das Arbeitsgedächtnis (d.h. die „onlineVerarbeitung“ von Informationen unter Beachtung verschiedener Details) als auch der freie Abruf aus dem Langzeitgedächtnis als besonders defizitär dar. Die visuell-räumlichen Gedächtnisleistungen werden nahezu als konsistent reduziert beschrieben und in einigen Untersuchungen gegenüber den Verballeistungen als dysproportional defizitär angegeben. Insgesamt finden sich diese Störungen bei Patienten mit einem chronischen Verlauf häufiger und sind in ihrer Qualität ausgeprägter. Die der nicht willentlichen Informationsverarbeitung unterliegenden, sog. nicht-deklarativen Gedächtnisleistungen (motorisches Lernen, sog. Priming-Phänomene) sind bei MS-Patienten bislang noch wenig erforscht, so dass anhand der bisher hierzu durchgeführten Untersuchungen nicht eindeutig entschieden werden kann, inwieweit in diesem Gedächtnisbereich bei MS-Patienten Leistungsdissoziationen nachzuweisen sind, die eine syndromatische Zuordnung der gefundenen Defizite im Sinne eines „subkortikalen“ vs „kortikalen“ Defizitprofils gestatten (10; vgl. aber hierzu 11). Wenngleich die Datenbasis hinsichtlich der Altgedächtnisleistungen bis dato ebenfalls gering ist, sprechen die wenigen hierzu durchgeführten Studien für diskrete retrograde Gedächtnisstörungen, sowohl für semantische als auch für autobiografische Inhalte, wobei, ähnlich der anterograden Gedächtnisdomäne, der Anteil an Patienten mit einem chronisch-progredienten Verlauf die markanteren Defizite aufweist (12).

\section{Alltagsrelevanz der MS-be- dingten Gedächtnisdefizite}

Wenngleich die Erforschung der neuropsychologischen Funktionsdefizite im Rahmen der Multiplen Sklerose in der letzten Dekade deutliche Fortschritte verzeichnen konnte, hat man erst jüngst die all- 
tagspraktischen Implikationen (z.B. Probleme im Erwerbsleben bei kognitiver Beeinträchtigung trotz erhaltener motorischer Fertigkeiten) dieser Störungen erkannt $(3,4)$. Gerade im Berufsleben ist die Entwicklung neuropsychologischer Defizite im Krankheitsverlauf für die individuelle Zukunftsplanung entscheidend. Somit sind neuropsychologische Befunde hinsichtlich der Arbeitsfähigkeit, aber auch bezogen auf die Lebensqualität sowie die Compliance, im Rahmen therapeutischer Bemühungen nicht nur im rehabilitativen Sektor, sondern auch im akut-neurologischen Bereich von besonderer Bedeutung. Basierend auf der in dieser Patientenpopulation häufigsten neuropsychologischen Beeinträchtigung des Gedächtnisses konnten wir in einer Reihe von klinisch-neuropsychologischen Studien auch anhand alltagsrelevanter Testverfahren (z.B. mittels Testmaterialien mit Alltagsbezug wie Portraits, Stadtpläne, Zeitungsmeldungen, Bedienung von Taschenrechnern etc.) differenzielle Leistungsbeeinträchtigungen bei MS-Patienten herausstellen (13). Damit konnten wir zunächst nachweisen, dass sich Störungen des Gedächtnisses bei MS-Patienten durchaus mittels alltagsrelevanter Verfahren abbilden lassen und die aus dem Einsatz solcher Tests gewonnenen Erkenntnisse des Weiteren unmittelbar einem therapeutisch-rehabilitativen Vorgehen nutzbar gemacht werden können. Zugleich wurde im Rahmen dieser Untersuchungen deutlich, dass sich die mnestischen Leistungen bei differenzierter Betrachtung der nach MS-Verlaufstyp getrennt analysierten Patientengruppen bei denjenigen Patienten als besonders defizitär herausstellten, die einen chronisch-progredienten Verlauf aufwiesen, während hingegen bei Patienten mit schubförmigen Verlauf eher allgemeine Kognitionsstörungen nachweisbar sind. Diese Befundkonstellation erforderte im weiteren Verlauf unserer Studien ein gesondertes Augenmerk bezüglich der Verlaufstypen und die zerebrale Läsionsausdehnung. Tatsächlich konnten wir herausstellen, dass die Patientengruppe mit ei- nem schubförmigen Verlauf die größte Varianz innerhalb des untersuchten kognitiven Leistungsspektrums aufwies. Weitere Untersuchungen machten wahrscheinlich, dass hierbei insbesondere das zerebrale Läsionsmuster als bestimmende Variable zu nennen ist (14). Schließlich führte uns die Zusammenschau der Befundkonstellationen zur Annahme eines „Schwellenmodells“, innerhalb dessen die gefundenen Defizitmuster in jeweiliger Abhängigkeit vom zerebralen Läsionsbefund zu interpretieren sind, d.h. diffus lokalisierte, multiple Läsionen führen zu unspezifischen kognitiven Störungen, die dann, wenn eine bestimmte „Läsionsschwelle" erreicht ist, immer auch zu mnestischen Leistungseinbußen führen. Aus dem beobachteten Läsionsverteilungsmuster schlugen wir schließlich vor, die bei MS-Patienten vorgefundenen neuropsychologischen Störungen als Folge einer multiplen, kortikalen Diskonnektion zu interpretieren (7).

\section{Das „multiple Diskonnekti- onssyndrom“}

Untersuchungen, die sich mit dem Zusammenhang zwischen Läsionsmuster und kognitivem Leistungsprofil bei MS beschäftigen, zeigen, dass extensive, vornehmlich periventrikulär lokalisierte Entmarkungsherde zwar zu einer globalen neuropsychologischen Leistungsreduktion führen, jedoch das Läsionsvolumen allein nicht für die spezifischen neuropsychologischen Symptome verantwortlich ist. Kritisch scheint hier vielmehr die Läsionsverteilung zu sein. Insbesondere kumulierte, multiple kortexnahe Demyelinisierungsherde, die sich neurologisch nicht funktionsbehindernd auswirken (sog. „silent lesions“), vermögen die kognitive Leistungsfähigkeit negativ zu beeinflussen, da ein räumlich disseminiertes und zeitlich aufeinander folgendes, kortexnahes Entmarkungsmuster langfristig zu multiplen Unterbrechungen kortiko-kortikaler Verbindungen führt und damit den netzwerkartigen Informationsverarbeitungsmodus des kognitiven Systemgefüges empfindlich stört (15).
Eine Zusammenschau der pathoanatomischen Daten aus der Literatur sowie eigene Untersuchungen zeigen, dass eine Kompensation offenbar nur bis zu einem bestimmten Läsionsausmaß möglich ist. Tatsächlich zeigt sich bei einem Vergleich der kognitiv-mnestischen Leistungen einzelner Subgruppen von MSPatienten, dass Patienten mit einem chronisch-progredienten Krankheitsverlauf deutlichere Gedächtnisstörungen sowie eine homogene, global-intellektuelle Leistungsbeeinträchtigung aufweisen, während bei Patienten mit einem schubförmigen Verlaufstyp eine große Leistungsvariabilität zwischen den Partialleistungen zu verzeichnen ist. In der folgenden Abbildung sind zwei typische, zerebrale Befallsmuster schematisch dargestellt. Unser Schwellenmodell erklärt die kognitiv-mnestischen Leistungsunterschiede der MS-Subtypen durch die unterschiedlichen zerebralen Affektionen (Abb. 1).

Großflächige, periventrikulärkonfluierende Läsionsmuster, die beim chronisch-progredienten Verlaufstyp nach langer Krankheitsdauer häufiger anzutreffen sind, führen durch die Affektion periventrikulär-limbischer sowie mesiotemporal lokalisierter (und damit gedächtnisrelevanter) Verarbeitungsschleifen sowohl zu mnestischen Störungen als auch zu einer allgemeinen intellektuellen Leistungsnivellierung. Vereinzelte Läsionen verursachen dagegen je nach Größe und Lokalisation (,strategische Läsionen“), im Einzelfall distinkte, neuropsychologische Teilleistungsstörungen, führen jedoch erst ab einer quantitativen „Schwelle“ zu einem generalisierten Leistungsdefizit. Vor diesem Hintergrund können die neuropsychologischen Defizite bei der MS nicht nur unter dem syndromatischen Begriff der „subkortikalen Demenz“ zusammengefasst, sondern beschreibend als Folge eines „multiplen Diskonnektionssyndroms“ aufgefasst werden. Die Annahme eines kumulativen Diskonnetionseffektes mit einer desintegrativen Wirkung auf funktionell relevante, neuronale Netzwerke bietet für die klinisch-neu- 
ropsychologisch vorgefundenen Symptomkonstellationen bei dieser Patientengruppe eine schlüssige Erklärung. Neben dem durch einen akuten Entzündungsprozess verursachten und das Läsionsausmaß bestimmenden Axon- und Myelinverlust ist auch der frühe Atrophiegrad für die Entwicklung der kognitiven Defizite entscheidend (16). Grundsätzlich wird das Läsionsausmaß im Rahmen konventioneller, T2- und T1-gewichteter MRT-Schichtaufnahmen eher unterschätzt. Durch die Anwendung spezieller MRT-Sequenzen und -Techniken (z.B. RARE und fasfFLAIR-Sequenzen, MR-Spektroskopie) kann inzwischen eine wesentlich reliablere Quantifizierung erfolgen $(15,16,17)$.

\section{Kognitive Defizite, Hirn- läsionen und Alltagsrelevanz}

Da kognitive Beeinträchtigungen sich bereits in der Frühphase einer MS manifestieren können, andererseits nur schwach mit dem körperlichen Behinderungsgrad korreliert sind, kann ein sensomotorisch unauffälliger Befund auf den ersten Blick von tatsächlich bestehenden Defiziten ablenken. Viel deutlicher scheint der Zusammenhang zwischen Läsionsmuster und kognitivem Leistungsprofil zu sein. Vor dem Hintergrund dieser Erkentnisse sollte eine kognitive Leistungseinschätzung frühzeitig erfolgen. Hierbei sollte die Diagnostik nicht defizitorientiert sein, sondern die aus diesen etwaigen Störungen resultierenden, alltagsrelevanten Problemfelder, mit dem Ziel einer therapeutischen Intervention isolieren. Tatsächlich konnte gezeigt werden, dass neuropsychologische Defizite bei MS-Patienten durch ein systematisches Funktionstraining erfolgreich rehabilitiert und/oder kompensiert werden können. Es ist zu erwarten, dass gerade die Alltagsrelevanz der geistigen Leistungsfähigkeit dazu führen wird, dass auch und gerade in der pharmakologisch orientierten Therapieforschung den kognitiven Indikatoren die Rolle von primären outcome-Parametern zugesprochen wird. Erste Ansätze wurden bereits im Zusammenhang mit der Erprobung neuer immunmodu- latorischer Substanzen erprobt. So konnten Fischer und Mitarbeiter mit einer Auswahl geeigneter neuropsychologischer Testverfahren zeigen, dass sich neben den klassischen neurologischen Parametern auch die kognitive Leistungsentwicklung unter einer zweijährigen Therapie mit Interferon- $\beta-1 \mathrm{a}$ stabilisieren ließ (18).

\section{Summary}

Multiple sclerosis (MS) is one of the most common neurological diseases of early and middle adulthood. Besides physical impairment MS bears also many psychosocial problems. Cognitive deficits appear in almost $50 \%$ of the patients in the course of the disease. Attention, memory and cognitive flexibility are predominantly affected. Theses disturbances may interfere with patients occupational status as well as social activities. Although the predictive validity of the cognitive abilities with respect to occupational demands is appreciated by a tantamount of actual studies only recently has thsi domain been considered in the general evaluation of the disease as well as a potential efficiency parameter in pharmacothereapeutic studies.

This paper focuses on the assessment and the everyday importance of the relevant deficits. Finally a functional-neuroanatomical model is introduced which might explain the organic basis of the cognitive deficits.

\section{Literatur}

1. De Sonneville LM, Boringa JB, Reuling IE et al. Information processing characteristics in subtypes of multiple sclerosis. Neuropsychologia 2002; 40: 1751-1765

2. Achiron A, Barak Y. Cognitive impairment in probable multiple sclerosis. Journal of Neurology, Neurosurgery and Psychiatry 2003; 74: 443-446

3. Amato MP, Ponziani G, Pracucci G, Bracco $L$ et al. Cognitive impairment in early-onset multiple sclerosis. Pattern, predictors, and impact on everyday life in a 4year follow-up. Archives of Neurology 1995; 52: 168-192

4. Amato, MP, Ponziani G, Siracausa G, Sorbi S. Cognitive dysfunction in early-onset multiple sclerosis. A reappraisal after 10 years. Archives of neurology 2001; 58: 1602-1606

5. Rao SM, Leo G], Bernardin L, Unverzagt F. Cognitive dysfunction in multiple sclerosis: I. Frequency patterns and prediction. Neurology 1991: 685-691
6. Hoogervorst ELJ, de Jonge $P$, Jelles B et al. The INTERMED: a screening instrument to identify multiple sclerosis patients in need of multidisciplinary treatment. Journal of Neurology, Neurosurgery and Psychiatry 2003; 74: 20-24

7. Calabrese P. Neuropsychologische Defizite bei entzündlichen Erkrankungen des ZNS. In W. Sturm, M. Herrmann \& C.-W. Wallesch (Hrsg.) Lehrbuch der klinischen Neuropsychologie. Lisse: Swets \& Zeitlinger Publishers, 2000.

8. Piras MR, Magnano I, Canu EDG et al. Longitudinal study of cognitive dysfunction in multiple sclerosis: neuropsychological, neuroradiological, and neurophysiological findings. Journal of Neurology, Neurosurgery and Psychiatry 2003; 74: 878-885.

9. Benedict RH, Bakshi R, Simon JH et al. Frontal cortex atrophy predicts cognitive impairment in multiple sclerosis. Journal of Neuropsychiatry and Clinical Neuroscience 2002; 14: 44-51

10. Rovaris M, Filippi M, Mincucci L et al. Cortical/subcortical disease burden and cognitive impairment in patients with multiple sclerosis. American Journal of neuroradiology 2000; 21: 402-408

11. Zarei M, Chandran S, Compston A, Hodges J. Cognitive presentation of multiple sclerosis: evidence for a cortical variant. Journal of Neurology, Neurosurgery and Psychiatry 2003; 74: 872-877

12. Beatty WW, Goodkin D, Monson N, Beatty PA, Hertsgaard D. Anterograde and retrograde amnesia in patients with chronic multiple sclerosis. Archives of Neurology 1988; 45: 611-619

13. Calabrese P, Haupts M, Babinsky R, Markowitsch HJ, Gehlen W. Alltagsgedächtnisleistungen bei Multipler Sklerose. Zeitschrift für Neuropsychologie 1993; 4: 4-16. 14. Calabrese P, Haupts M, Gehlen W. Verlaufsabhängige Gedächtnisstörungen und Läsionsmuster bei multipler Sklerose. Neurologie und Rehabilitation 2000; 6: 184-188

15. Lazeron LHC, Langdon DW, Filippi M et al. Neuropsychological impairment in multiple sclerosis patients: the role of (juxta)cortical lesions on FLAIR. Multiple Sclerosis 2000; 6: 280-285.

16. Zivanidov R, Sepcic J, Nasuelli D et al. A longitudinal study of brain atrophy and cognitive disturbances in the early phase of relapsing-remitting multiple sclerosis. Journal of Neurology, Neurosurgery and Psychiatry 2001; 70: 773-780

17. Christodolou C, Krupp LB, Liang Z et al. Cognitive performance and MR markers of cerebral injury in cognitively impaired MS patients. Neurology 2003; 60: 1793-1798. 18. Fischer JS, Priore RL, Jacobs LD et al. Neuropsychological effects of interferon $\beta$ 1 -a in relapsing-remitting multiple sclerosis. Annals of Neurology 2000; 48: 885-892

\section{Korrespondenzadresse:}

Dr. Dipl.-Psych. Pasquale Calabrese Neurologische Universitätsklinik Bochum Bereich Neuropsychologie In der Schornau 23-25

44829 Bochum 